\title{
The effects of probiotic supplementation on gut microbiota composition, immunological and biochemical markers in healthy volunteers
}

\author{
A. O'Connor ${ }^{1}$, A. O’Sullivan ${ }^{1}$, S. Kaluskar ${ }^{1}$, B. Mion ${ }^{1}$, J. Marchesi ${ }^{2}$, K. Cashman ${ }^{3}$, A. Flynn ${ }^{3}$, \\ F. Shanahan ${ }^{2}$, L. Brennan ${ }^{1}$ and M. J. Gibney ${ }^{1}$ \\ ${ }^{1}$ UCD Institute of Food and Health, School of Agriculture, Food Science and Veterinary Medicine, Veterinary Science \\ Centre, Belfield, Dublin 4, Republic of Ireland, and ${ }^{2}$ Alimentary Pharmabiotic Centre and ${ }^{3}$ Department of Food \& \\ Nutritional Sciences, University College Cork, National University of Ireland, Cork, Republic of Ireland
}

Lactobacillus and Bifidobacterium strains are the most-commonly-used probiotics and are thought to affect immune function. Many welldesigned studies have provided evidence for the use of these strains in managing gastrointestinal disorders ${ }^{(1)}$, prevention of atopy in infants $^{(2)}$ and also colon cancer in men ${ }^{(3)}$. The potential immunological effects of probiotics in healthy individuals has been cited ${ }^{(4)}$. The aim of the present study was to determine the ability of Lactobacillus salivarius UCC 118 to colonize the large intestine, modify total gut microbiota composition and measure its effects on a number of immunological markers.

A double-blinded placebo-controlled dietary intervention was carried out in two centres (University College Dublin and University College Cork). Healthy volunteers were randomised to one of four treatment groups, according to age and gender. The four treatment groups were defined as follows: group 1, daily cholecalciferol $(15 \mu \mathrm{g})$ and probiotic (Lactobacillus salivarius $10^{9}$ colony-forming units (cfu) $/ 5 \mathrm{~g}$ sachets suspended in maltodextrin); group 2, daily cholecalciferol and placebo probiotic (maltodextrin); group 3, daily vitamin D placebo and probiotic; group 4, daily vitamin D placebo and probiotic placebo. The cholecalciferol and matching placebo was consumed in capsule form, while the probiotic and the probiotic placebo (in powder form) were mixed with milk for consumption. Fasting urine, blood and faeces samples were collected before and after the 4-week intervention period. Food records ( $3 \mathrm{~d})$ estimated habitual diet and were also used to monitor intervention compliance. From the faecal samples bacterial DNA was extracted. Changes in Lactobacillus and total bacteria communities were measured by denaturing gradient gel electrophoresis (DGGE) using lactic acid bacteria and universal 16S-rRNA primers respectively. Additionally, PCR using strain-specific primers for L. salivarius UCC 118 was carried out. Specific bands were excised from the gels and sequenced. IL-6, TNF $\alpha$ and C-reactive protein were measured in pre- and post-intervention serum samples by ELISA and glucose was measured by a colorimetric assay.

Treatment groups did not significantly differ in relation to age, gender or BMI. Results from DGGE profiles of Lactobacillus community and strain-specific PCR suggest that the probiotic was established in the gut of 55.5\% of volunteers that consumed the probiotic and from which a PCR product could be obtained. Pairwise comparison of post-intervention DGGE profiles for the total bacterial community showed significant changes $(P<0.05)$ in the gut microbiota following probiotic consumption. Serum IL-6 levels were $0.13(\mathrm{sE} 0.02) \mathrm{pg} / \mathrm{ml}$ at baseline and $0.11(\mathrm{SE} 0.01) \mathrm{spg} / \mathrm{ml}$ at end point following probiotic intervention. Serum TNF $\alpha$ levels were 164 (SE 38.7) pg/ml at baseline and 190 (SE 39.1) at end point following probiotic intervention. Serum C-reactive protein levels were 1.78 $(\mathrm{SE} 0.24) \mathrm{mg} / \mathrm{l}$ at baseline and $1.57(\mathrm{SE} 0.21) \mathrm{mg} / \mathrm{l}$ at end point following probiotic intervention. Fasted serum glucose levels were 5.11 (SE 0.05 ) $\mathrm{mmol} / \mathrm{l}$ at baseline and 5.05 (SE 0.05 ) $\mathrm{mmol} / \mathrm{l}$ following probiotic intervention. No significant difference in serum levels of IL-6, $\mathrm{TNF} \alpha$ and C-reactive protein were observed following probiotic consumption. However, general linear model analysis showed an interaction between gender and serum glucose levels in females following probiotic consumption $(P<0.05)$.

In conclusion, supplementation with $1 \times 10^{9} \mathrm{cfu}$ Lactobacillus salivarius UCC118 for $28 \mathrm{~d}$ in healthy volunteers significantly altered gut microbiota composition and no effect on the immunological markers measured was observed.

1. Guandalini S, Pensabene L, Dias MA et al. (2000) J Paediatr Gastroenterol Nutr 30, 54-60.

2. Kalliomaki M, Salminen S, Poussa T et al. (2003) Lancet 361, 1869-1871.

3. Hatakka K, Holma R, El-Nezami H et al. (2008) Int J Food Microbiol 128, 406-410. 